\title{
Bioluminescence ATP monitoring as a surrogate marker for microbial load on hands and surfaces in the home
}

\author{
Elaine L. Larson ${ }^{\mathrm{a}, *}$, Allison E. Aiello ${ }^{\mathrm{b}}$, Cabilia Gomez-Duarte ${ }^{\mathrm{c}}$, Susan X. Lin ${ }^{\mathrm{c}}$, \\ Lillian Lee ${ }^{\mathrm{d}}$, Phyllis Della-Latta ${ }^{\mathrm{e}}$, Charlotte Lindhardt ${ }^{\mathrm{f}}$ \\ ${ }^{a}$ Schools of Nursing and Public Health, Columbia University, 630 W. 168th Street, New York, NY, USA \\ ${ }^{\mathrm{b}}$ School of Public Health, Columbia University, New York, NY, USA \\ ${ }^{\mathrm{c}}$ School of Nursing, Columbia University, New York, NY, USA \\ ${ }^{\mathrm{d}}$ Clinical Microbiology Laboratory, New York Presbyterian Hospital, New York, NY, USA \\ ${ }^{\mathrm{e}}$ Department of Pathology, College of Physicians and Surgeons, Columbia University, New York, NY, USA \\ ${ }^{\mathrm{f}}$ Merck Chemicals Ltd., Wales, UK \\ Received 16 November 2002; accepted 16 March 2003
}

\begin{abstract}
To evaluate the validity and reliability of adenosine triphosphate (ATP) monitoring as a surrogate marker for cleanliness, the kitchen table surface in 225 inner-city homes was sampled by microbiologic culture and by two types of biomass monitoring systems (HY-LiTE 2 ATP System and HY-RiSE Colour Hygiene Test Strip, EM Science, Gibbstown, New Jersey, USA). A randomly selected hand of the homemaker $(n=225)$ was also cultured and sampled with the ATP monitoring system immediately after handwashing. Log microbial counts on hands ranged from 3.2 to 7.0 and from the table, 1.0 to 5.5. While the traditional ATP readings (HY-LiTE) and the color strips were significantly correlated $(R=0.18, P=0.01)$, there was no significant correlation between the ATP monitor readings and the colony-forming units counts on either the hands $(R=-0.03, P=0.62)$ or the table $(R=0.04, P=0.58)$. Such biomass measurements are not a substitute for quantitation of microbial load.

(C) 2003 Elsevier Ltd. All rights reserved.
\end{abstract}

Keywords: ATP bioluminescence; Biomass monitoring; Handwashing; HACCP; Hygiene

\section{Introduction}

The inanimate environments of homes and healthcare facilities harbor niches which support the growth of large numbers of micro-organisms. While household and outpatient transmission of a variety of infectious diseases has been demonstrated, particularly for diseases spread by the fecal-oral and respiratory routes, there are few data which link specific levels of ambient microbial contamination with increased risk of disease transmission. Further elucidation of any relationship between environmental microbial contamination and risk of infectious disease transmission is vital to planning effective prevention and control strategies. One of the challenges to developing such strategies is the practical

\footnotetext{
*Corresponding author. Tel.: + 1-212-305-0723; fax: + 1-212-3050722.

E-mail address: ell23@columbia.edu (E.L. Larson).
}

and economic constraints, which prevent researchers from obtaining microbial data in a timely fashion and linking that to home hygiene practices.

Recently, a group of investigators used a rapid system to measure adenosine triphosphate (ATP) by means of bioluminescence (HY-LiTE ${ }^{\mathrm{TM}}$, EM Science, Gibbstown, New Jersey, USA) as a surrogate marker to assess the quality of handwashing in a 1200-bed teaching hospital (Lodola et al., 1999; Marena et al., 2002). This system employs ATP bioluminescence as a rapid indicator of levels of biologic material present on surfaces or in liquids. It is currently used extensively in the food service industry as an indicator of the bioload on surfaces and in foods.

In a previous preliminary study conducted by this investigative team under controlled laboratory conditions using 60 repetitive tests with Staphylococcus aureus and Salmonella choleraesuis, we found significant correlations between bacterial counts and ATP readings 
(Larson et al., 2001), which led us to pursue the application of this technology in a community setting. Therefore, the aim of this study was to evaluate the validity and reliability of using a rapid ATP monitoring system as a surrogate marker for levels of cleanliness on an environmental surface and on hands in the home. Specific objectives of the study were to examine the correlation between microbial counts and ATP measurements on hands and an environmental surface, and to assess the potential application of ATP technology as an indicator of the effectiveness of standard cleaning procedures for hands and environmental surfaces.

\section{Materials and methods}

\subsection{Setting and scope}

The study was conducted in an upper Manhattan neighborhood comprised primarily of multiple dwelling apartment complexes. The neighborhood was selected by convenience as part of a larger clinical trial to examine the role of home hygiene practices on the incidence of infectious disease symptoms because it is densely populated with many households including several generations, often with young children, living in close proximity. Participants were recruited by posters, brochures and word-of-mouth in local pediatric clinics, community centers, churches, and posters placed on local bulletin boards. To qualify for the study, households had to include at least three individuals, at least one being a preschool child, had to be conversant in either Spanish or English, and willing to allow the research staff to make a home visit. Initially, 238 households were enrolled, and 225 households were available for the ATP testing and microbiologic sampling.

\subsection{ATP measurement}

ATP measurement has been used as an indicator of hygiene, i.e. the absence of filth, which might include bacteria, other microbes, dirt, feces, food, etc. Bioluminescence is a biologic reaction that produces light, as in fireflies. When ATP and luciferin/luciferase enzyme complex combine, a reaction which produces light occurs. The quantity of light is expressed in relative light units (RLU), which directly correlates with the amount of ATP present and thus with the level of biologic load on the sampled area (Deshpande, 2001).

Two sites were tested - the eating surface of the household's kitchen table and the hand of the homemaker. Two monitoring systems were used to obtain biomass readings from the table: HY-LiTE ${ }^{\text {TM }} 2$ ATP System and HY-RiSE Colour Hygiene Test Strip, EM Science, Gibbstown, New Jersey, USA). The former entails sampling a $4 \times 4 \mathrm{~cm}^{2}$ surface area with a sampling pen and entering the data into a data logger. "Pass" rates and "fail" rates have typically been set in the food service industry for foods (ranging from 30 to 500 RLU). The Test Strip measures NAD $/ \mathrm{NADH}^{+}$and $\mathrm{NADP} / \mathrm{NADPH}^{+}$with a simplified procedure in which the sampling strip is placed on a test surface and reagents are then applied which result in a yellow-topurple reaction visually rated on a scale of $0-6$. The two different measurement devices both measure energy transport molecules in the respiratory chain in all living organisms. For the hands, only the ATP monitoring system, but not the strips, were used.

Samples were obtained by one of four trained data collectors. A consultant from the manufacturer of the ATP bioluminescence monitor (Merck Chemicals Ltd., UK) made two 2-day site visits to train staff. This training included return demonstrations and on-site home visits to assure that each data collector was proficient in the techniques. The table sample was obtained with a specially supplied swab according to the instruction manual from the manufacturer. Results were printed out and entered into the machine's database automatically. Samples from the hands were obtained by dipping another swab supplied by the manufacturer into the sampling solution used to obtain the microbiologic hand culture, and the ATP reading was obtained, read, and recorded in the same manner as the environmental sample.

\subsection{Hand culture technique}

Hand cultures were obtained during a home visit immediately after handwashing. Participants were asked to wash their hands in their usual manner and then dry them with a clean paper towel. The hand to be cultured (right or left) was randomly selected by the data collector by flipping a coin. A modified glove-juice technique was used whereby the subject inserted the hand into a sterile polyethylene bag containing $50 \mathrm{ml}$ of sampling solution $(0.075 \mathrm{M}$ phosphate buffer, $\mathrm{pH} 7.9)$. This solution disperses the macrocolonies into single cells for quantitation. The entire hand was massaged through the wall of the bag for $1 \mathrm{~min}$. Samples from the kitchen table were obtained with a rayon-tipped swab (Culturette Systems, Baxter Healthcare Corporation, Deerfield, Illinois, USA) moistened with transport medium. Data collectors swabbed a surface on the table immediately adjacent to the location of the ATP measurement, using a $4 \times 4$ in $(10.2 \mathrm{~cm})$ template, rotating the swab with pencil eraser-type pressure for $1 \mathrm{~min}$.

As with the ATP measurement protocol, data collectors were trained in the microbiologic sampling techniques and demonstrated competence and consistency in the presence of the coinvestigators before obtaining samples. The hand and table samples for microbiologic culture were obtained during the same 
home visit, and were transported to the laboratory and processed within a few hours of sampling.

Samples were diluted 10 fold, up to $10^{-3}$, and spread plated onto sheep blood agar (5\%), MacConkey, colistin-nalidixic acid, and Sabouraud's with chloramphenicol and gentamicin and bile esculin (Becton Dickinson Microbiology Systems, Sparks, Maryland, USA), incubated at $35^{\circ} \mathrm{C}$ and observed daily for growth over $48 \mathrm{~h}$ for bacteria and up to 7 days for yeast. Total counts were calculated from the blood agar plates.

\subsection{Procedures}

The study was approved by the New York Presbyterian Medical Center Institutional Review Board, and each participant signed a written consent document. Samples were obtained by the trained interviewers during a single home visit of about $1 \mathrm{~h}$ in duration. For quality control, the study's Project Director made random site visits on $10 \%$ of the home visits with each data collector to assure consistency and accuracy in data collection procedures.

\subsection{Data analysis}

Counts of colony-forming units (cfu) and RLU are not normally distributed, and were therefore converted to $\log _{10}$ to normalize the data for analyses. The Pearson (for HY-LiTE, which is continuous data) or Spearman (for Test Strip, which is ordinal data) correlation coefficients were calculated to compare readings and cfu for each paired test for the hands and the table surfaces. A one-way analysis of variance was used to compare mean differences in cfu counts with the Color Test Strip readings on the table. To determine the correlation between cfu and RLU at various levels of contamination, both ATP and cfu readings were divided into quartiles (lowest $25 \%, 26$ th-50th percentile, 5175th percentile, highest $25 \%$ ), and the quartiles compared using $\chi^{2}$ analyses. Bland and Altman (1986) have suggested that correlation coefficients may not be the best indicator of agreement for comparing a new measurement technique with an established one. They suggested that the precision of the estimate could be improved by plotting the differences between the two measures against the mean of the two measures, so we therefore followed this procedure as well. Finally, because the limits of detection of microbial growth in some ATP monitoring systems has been reported to be about $10^{4} \mathrm{cfu} / 100 \mathrm{~cm}^{2}$ (Corbitt et al., 2000; Davidson et al., 1999), correlational analyses were repeated for only those table and hand samples with microbial counts $>10^{4}$.

\section{Results}

Microbial data were available from 225 hand and 219 table samples; ATP readings were available for 221 hands and tables. Log microbial counts on hands ranged from 3.2 to 7.0 and from the table, 1.0 to 5.5. Log ATP readings on hands ranged from 0.7 to 4.9 (5-76000) RLU and from table, 0.8 to 4.6 (15-44 000) RLU. Color strip readings on the table ranged from 0 to 3 . While the traditional ATP readings (HY-LiTE) and the color strips were significantly correlated $(R=0.16, P=0.03)$, there was no significant correlation between the ATP monitor readings and the cfu counts on either the hands ( $R=0.09, P=0.17)$ or the table $(R=0.05, P=0.45)$, see Table 1. There was no significant correlation between hand or table cfu and ATP counts when they were divided into four quartiles $(P=0.82$ and 0.32 , respectively), nor were there significant differences in mean cfu counts by color strip reading $(P=0.75)$. Plotting the differences against the means of the RLU and cfu did not improve the correlations, since the Bland and Altman (1986) analysis showed the bias between RLU and cfu was $2.28 \operatorname{logs}$ and the limits of agreement ranged from $0.035(95 \%$ confidence limits (CL): $-3.6,4.27)$ and 4.23 (95\% CL: 0.29-8.16). Limiting the analysis to only those samples with counts $>10^{4}$ also did not improve the correlation.

\section{Discussion}

ATP abounds in most biological niches including body secretions, food, etc., and ATP levels have been shown in some studies to correlate well with levels of hygiene. Nevertheless, in this series of tests there was no correlation with either method of biomass measurement and the microbial counts on hands or tables. Murphy

Table 1

Microbiologic and ATP results from kitchen table $(n=219)$ and hands $(n=225)$

\begin{tabular}{lllll}
\hline Sampling site & Mean log cfu $( \pm$ s.d. $)$ & $\begin{array}{l}\text { Mean log ATP reading in } \\
\text { relative light units (monitor) }\end{array}$ & $\begin{array}{l}\text { Mean reading (strip) range: } \\
0-6\end{array}$ & $\begin{array}{l}\text { Correlation coefficient } \\
(P \text { value })^{*}\end{array}$ \\
\hline Table & $2.97(1.00)$ & $2.61(0.75)$ & $2.22(0.85)$ & $0.05(p=0.45)$ \\
Hand & $4.90(0.64)$ & $2.63(0.79)$ & Not performed & $0.09(p=0.17)$ \\
\hline
\end{tabular}

*Pearson's correlation between log cfu and monitored ATP counts. 
et al. (1998), for example, compared ATP monitoring with swabbed contact samples of surfaces in four milk plants and reported a reasonable correlation between low, medium, and high microbial counts and ATP readings. In $>300$ samples from poultry carcasses, the correlation coefficient between ATP tests and microbiology was 0.82 (Siragusa et al., 1996). Griffith et al. (2000) used both microbiological and ATP bioluminescence to evaluate hospital cleaning regimens. Although they did not report correlation coefficients between the two, ATP and microbial counts were both highest in the kitchen samples, but there was considerable variation in both. Some studies have simply reported RLU counts as a measure of hygiene, but have not provided data regarding sensitivity, specificity or reproducibility of their findings (Nijskens and Baakman, 2001).

There may be several reasons why we failed to find a correlation between RLU and cfu in this study. One limitation of ATP bioluminescence measurement is that it detects ATP generically and cannot differentiate the source. Although some progress has been made to segregate microbial from non-microbial ATP, ATP from viable microbes cannot be distinguished from ATP from dead microbes or higher organisms. Trials in meat processing and production plants have shown this monitor to be more sensitive and reliable for tracking surface contamination than microbial sampling (deZutter et al., 1998), since the monitor quantitates surface ATP rather than microbial growth per se. For example, ATP monitoring has been shown to be particularly useful in detecting the presence of saliva on dental equipment (Douglas and Rothwell, 1991), and the presence of suspended solids in sludge interferes with bioluminescence tests (Chu et al., 2001). Thus, the presence of any ATP will increase the RLU levels, regardless of the microbial count. Clearly, on surfaces in the home environment and on hands, ATP is present from sources other than microbes.

A second reason why ATP readings and microbial counts might not correlate is that the limits of detection of microbial growth in some ATP monitoring systems has been reported to be about $10^{4} \mathrm{cfu} / 100 \mathrm{~cm}^{2}$ (Corbitt et al., 2000; Davidson et al., 1999). This was not the case in this study, however, because microbial counts were generally higher than the detectable limit, and since limiting the analysis to only those samples with counts $>10^{4}$ did not improve the correlation.

Studies have often reported considerable variation in both ATP readings and cfu counts (Griffith et al., 2000). Whereas some have reported less variability with ATP monitoring (Davidson et al., 1999), Tebbutt (1999) concluded that since ATP monitoring demonstrated such large variability it could not accurately predict the level of bacterial contamination on cutting boards.

Marena et al. (2002) reported a significant correlation between ATP readings and cfu on hands, also using the
HY-LiTE machine $(r=0.68, P<0.001)$. Even with this high correlation, however, about one-third of ATP/cfu pairs were not concordant for assessing "clean" or "dirty" sites, as defined by cutoffs set by the authors. In their study, both the ATP and the cfu measurements were obtained by swabbing the surface of the hand rather than using the glove-juice technique and the ATP sampling protocol recommended by the manufacturers. Certainly, findings will vary, depending upon data collection procedures, but since we used well-established methods for data collection, this difference probably does not explain our lack of correlation.

Finally, it is possible that ATP bioluminescence monitoring should not be used as a predictor of microbial contamination as some have claimed. Poulis et al. (1993) noted that there was a poor relationship between ATP readings and conventional microbiological culture methods in samples from food processing facilities. Colquhoun et al. (1998) compared three different ATP hygiene monitoring systems and found that they differed considerably in sensitivity and reproducibility.

In summary, ATP monitoring has been widely used, particularly in the food service industry, as a rapid screening method for measuring gross, general levels of cleanliness, i.e. the presence of organic matter, but it is not a reliable substitute for culturing in situations in which a measurement of microbial contamination is needed nor for correlating risk of infectious disease transmission. ATP bioluminescence should not be used as a substitute for quantitation of microbial load.

\section{Acknowledgements}

This work was supported by 1R01NR05251-01, "Home Hygiene Practices and Infection Transmission in Households", National Institutes of Health, National Institute for Nursing Research.

\section{References}

Bland, J.M., Altman, D.G., 1986. Statistical methods for assessing agreement between two methods of clinical measurement. Lancet 1 (8476), 307-310.

Chu, C.P., Lee, D.J., Chang, B.V., Liao, C.S., 2001. Using ATP bioluminescence technique for monitoring microbial activity in sludge. Biotechnol. Bioeng. 75 (4), 469-474.

Colquhoun, K.O., Timms, S., Fricker, C.R., 1998. A simple method for the comparison of commercially available ATP hygienemonitoring systems. J. Food Prot. 61 (4), 499-501.

Corbitt, A.J., Bennion, N., Forsythe, S.J., 2000. Adenylate kinase amplification of ATP bioluminescence for hygiene monitoring in the food and beverage industry. Lett. Appl. Microbiol. 30 (6), 443-447.

Davidson, C.A., Griffith, C.J., Peters, A.C., Fielding, L.M., 1999. Evaluation of two methods for monitoring surface cleanliness-ATP 
bioluminescence and traditional hygiene swabbing. Luminescence 14 (1), 33-38.

Deshpande, S.S., 2001. Principles and applications of luminescence spectroscopy. Crit. Rev. Food Sci. Nutr. 41 (3), 155-224.

deZutter, L., Hellwig, K., Lindhardt, C., 1998. ATP method is highly suitable for hygiene monitoring. DeKeurmeester 3, 5-10.

Douglas, C.W., Rothwell, P.S., 1991. Evaluation of a hygiene monitor for detection of contamination in dental surgeries. Br. Dent. J. 170 (9), 331-335.

Griffith, C.J., Cooper, R.A., Gilmore, J., Davies, C., Lewis, M., 2000. An evaluation of hospital cleaning regimes and standards. J. Hosp. Infect. 45 (1), 19-28.

Larson, E.L., Gomez-Duarte, C., Qureshi, K., Miranda, D., Kain, D.J., Cablish, K.L., 2001. How clean is the home environment? A tool to assess home hygiene. J. Community Health Nurs. 18 (3), $139-150$.

Lodola, L., Marena, C., Olivieri, P., 1999. A prospective crossover study for assessing handwashing practice at San Matteo Hospital. Preliminary results after two months. Paper Presented at the Ninth Annual Conference of The Society for Healthcare Epidemiology of America, San Francisco, CA.

Marena, C., Lodola, L., Bulgheroni, A., Carretto, E., Zecca, M., Maserati, R., Zambianchi, L., 2002. Assessment of handwashing practices using chemical and microbiological methods: preliminary results from a prospective study. Am. J. Infect. Control 30 (6), 334-340.

Murphy, S.C., Kozlowski, S.M., Bandler, D.K., Boor, K.J., 1998. Evaluation of adenosine triphosphate-bioluminescence hygiene monitoring for trouble-shooting fluid milk shelf-life problems. J. Dairy Sci. 81 (3), 817-820.

Nijskens, J.J., Baakman, R., 2001. [Performance indicators of hygiene during animal transportation. Results of a large scale monitoring of trucks with ATP-bioluminescent technique]. Tijdschr Diergeneeskd 126 (14-15), 488-490.

Poulis, J.A., de Pijper, M., Mossel, D.A., Dekkers, P.P., 1993. Assessment of cleaning and disinfection in the food industry with the rapid ATP-bioluminescence technique combined with the tissue fluid contamination test and a conventional microbiological method. Int. J. Food Microbiol. 20 (2), 109-116.

Siragusa, G.R., Dorsa, W.J., Cutter, C.N., Perino, L.J., Koohmaraie, M., 1996. Use of a newly developed rapid microbial ATP bioluminescence assay to detect microbial contamination on poultry carcasses. J. Biolumin. Chemilumin. 11 (6), 297-301.

Tebbutt, G., 1999. Comparison of traditional and rapid methods for assessing the risk of bacterial cross-contamination from cutting boards. Int. J. Environ. Health Res. 9 (1), 67-74. 